\title{
Prevalência e impacto da incontinência urinária na qualidade de vida da mulher
}

Ana Isabel Silva, ${ }^{1}$ Carla Almeida, ${ }^{2}$ Hélder Aguiar, ${ }^{3}$ Margarida Neves, ${ }^{4}$ Maria João Teles $^{5}$

\section{RESUMO}

Objetivos: A incontinência urinária é um problema de saúde com repercussões multidimensionais que interferem negativamente na qualidade de vida das mulheres. É frequentemente subdiagnosticada e não tratada. Neste sentido, pretende-se determinar a prevalência da incontinência urinária, avaliar o impacto desta patologia na qualidade de vida e a sua eventual associação com fatores epidemiológicos.

Tipo de Estudo: transversal analítico.

Local: unidades funcionais onde trabalham os autores.

População: amostra de 1.918 mulheres com mais de 40 anos.

Métodos: As variáveis estudadas foram: idade, estado civil, escolaridade, índice de massa corporal, número de filhos, tipo de incontinência, duração, referência do problema ao médico e sua orientação. $O$ impacto na qualidade de vida foi avaliado através do questionário CONTILIFE@. A análise estatística foi realizada pelo programa SPSS $20.0^{\circledR}$. Usaram-se como testes paramétricos o teste t Student e a correlação de Pearson e como teste não paramétrico o método de Spearman. Adotou-se um nível de significância estatística de $p \leq 0,05$.

Resultados: Das 1.291 mulheres inquiridas (idade média 60 anos), 23\% tinham incontinência e a sua qualidade de vida global foi diminuída para 6,7 (em 10). O impacto na qualidade de vida aumenta $(p \leq 0,05)$ com o índice de massa corporal e com o número de partos. Das $38 \%$ mulheres com incontinência que abordaram esta questão com o seu médico de família (MF), $66 \%$ tiveram uma orientação (farmacológica ou outra, como referenciação). A abordagem do problema com o MF associou-se de forma direta às mulheres com maior impacto negativo $(p<0,001)$ na qualidade de vida.

Conclusão: Este estudo demonstra que, embora tendo uma prevalência elevada e com forte impacto na qualidade de vida, apenas cerca de $1 / 3$ das mulheres com incontinência urinária aborda esta problemática com o seu médico, sendo que $2 / 3$ obtêm uma orientação - farmacológica ou outra como referenciação.

Palavras-chave: Incontinência Urinária; Mulher; Qualidade de Vida.

\section{INTRODUÇÃO}

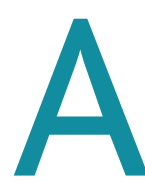
definição de incontinência urinária (IU) como qualquer perda involuntária de urina (no último ano ou mês) foi introduzida pela Internacional Continence Society, com o intuito de uniformizar os estudos epidemiológicos, atendendo a que até então o conceito de IU era variável e dependente das diferentes culturas, países, estilos de vida, género e idade. ${ }^{1,2}$

'Interna de MGF, USF Nova Salus, 'Interna de MGF, USF Famílias, ${ }^{3}$ Interno de MGF, USF Vale do Vouga, ${ }^{4}$ Interna de MGF, USF Calâmbriga, I'Interna de MGF, USF Camélias.
A IU é mais frequente em mulheres que em homens e aumenta com a idade e a sua prevalência na população feminina, de acordo com os diversos estudos existentes, é muito variável (5-69\%)., ${ }^{3,45}$ Essa variabilidade ocorre devido à escolha da amostra, em função da idade, género, nível cultural, gravidade da doença, tipo de IU e sua baixa notificação.

Segundo alguns investigadores, a IU constitui um problema de saúde que pode ter repercussões físicas, socioeconómicas e psicológicas consideráveis. ${ }^{5-9}$

Assim, nos últimos anos, tem havido um crescente desenvolvimento de estudos que se baseiam, não ape- 
nas nos sintomas da IU, mas essencialmente, na sua interferência na qualidade de vida (QdV) das mulheres. ${ }^{7}$ De modo a determinar esse impacto, foram desenvolvidos vários instrumentos de avaliação de QdV em mulheres com IU. ${ }^{7,10,11}$ Tanto quanto os investigadores sabem, a nível nacional, não existe ainda nenhum estudo sobre o impacto da QV em mulheres, que conste em alguma publicação indexada.

Apesar do impacto da IU na vida das mulheres ser substancial, assim como a sobrecarga económica imposta à sociedade, continua a ser um problema pouco reconhecido e tratado. ${ }^{12,13}$ Por um lado, a maioria das doentes não procura ajuda médica por vergonha, medo da cirurgia, pela ideia errónea de que se trata de um problema intratável e é uma inevitável consequência do envelhecimento ou da história de partos vaginais prévios ou pela ideia que a IU pode precipitar a sua institucionalização. ${ }^{5,9,11}$

Por outro lado, os próprios profissionais de saúde contribuem de igual forma para o subdiagnóstico e subtratamento. De facto, a Organização Mundial de Saúde chamou à IU «o derradeiro tabu médico». Os fatores que contribuem para isto são: medo de invasão da privacidade e dignidade da paciente, ao questionar esta problemática; défice de formação e informação acerca do diagnóstico e tratamentos disponíveis; falsa premissa de que os tratamentos são fúteis e dependentes de fatores psicológicos da própria utente; receio dos efeitos laterais das opções terapêuticas; IU encarada como uma consequência inevitável da idade., ${ }^{6,9}$

Este trabalho de investigação tem como objetivos primários: avaliar, numa população portuguesa essencialmente urbana de mulheres com idade superior a 40 anos, a prevalência da IU e o seu impacto na QdV; como objetivos secundários, os autores pretendem correlacionar o impacto da IU na QdV com fatores sociodemográficos, com o tipo de IU, com a referência da IU como problema de saúde ao médico de família (MF) e com o estabelecimento de orientação para o tratamento da IU.

\section{MÉTODOS}

Trata-se de um estudo observacional, transversal e analítico que decorreu entre julho de 2011 e setembro de 2012, nos locais de trabalho dos seus investigadores: Unidade de Saúde Familiar (USF) Camélias e Nova Salus [Agrupamentos de Centros de Saúde (ACeS) Grande Porto VII], USF Famílias (ACeS Entre Douro e Vouga
I) e USF Calâmbriga e Vale do Vouga (ACeS Entre Douro e Vouga II).

A população do estudo incluiu mulheres com idade igual ou superior a 40 anos, inscritas e com MF nas USF que constituem local de trabalho dos investigadores.

Os critérios de exclusão considerados pelos autores são: ausência de contacto telefónico válido no sistema informático SINUS; doenças neuropsiquiátricas que comprometam a capacidade cognitiva, codificadas no sistema informático SAM, de acordo com a ICPC-2 (International Classification of Primary Care) - códigos: P70 (Demência); P71 (Psicose orgânica); P72 (Esquizofrenia); P98 (Psicose não especificada); P99 (Perturbação psicológica, outra); N80 (Lesão craniana); N85 (Malformação congénita neurológica); institucionalizadas, internadas ou acamadas aquando do momento da seleção; algaliadas cronicamente; grávidas, no momento da seleção ou no ano anterior; emigradas; falecidas ou que não falassem ou entendessem a língua portuguesa.

O tamanho amostral foi determinado através do programa Epi Info ${ }^{\mathrm{TM}} 3.5 .2$, considerando-se uma prevalência de IU esperada de $30 \%$, com um nível de precisão de $5 \%$, um intervalo de confiança de $95 \%$ e uma taxa de não resposta de $30 \%$. A prevalência de IU esperada foi baseada num estudo de prevalência realizado em Portugal a nível dos Cuidados de Saúde Primários (CSP). ${ }^{24}$ Assim, para uma população de 18.968, foi obtida uma amostra de 1.918 mulheres.

Para a seleção da amostra recorreu-se a uma técnica de amostragem aleatorizada de forma simples sem reposição através do programa informático RANDOM.ORG®, disponível no sítio de internet http: //www.random.org.

Após seleção, todas as utentes foram contactadas telefonicamente e, após uma breve apresentação e contextualização, foi colocada a seguinte questão: «A senhora teve alguma perda involuntária de urina (ou seja, sem o seu controlo), no último mês?». Em caso de resposta afirmativa, a mulher foi convidada a se deslocar à sua USF, onde posteriormente assinou o consentimento informado e onde lhe foi aplicado um questionário, presencialmente. O processo de seleção da amostragem encontra-se discriminado na Figura 1.

A definição e classificação da IU adotadas foram as mesmas descritas pela International Continence Society, em que a IU equivale a «qualquer perda involuntária 


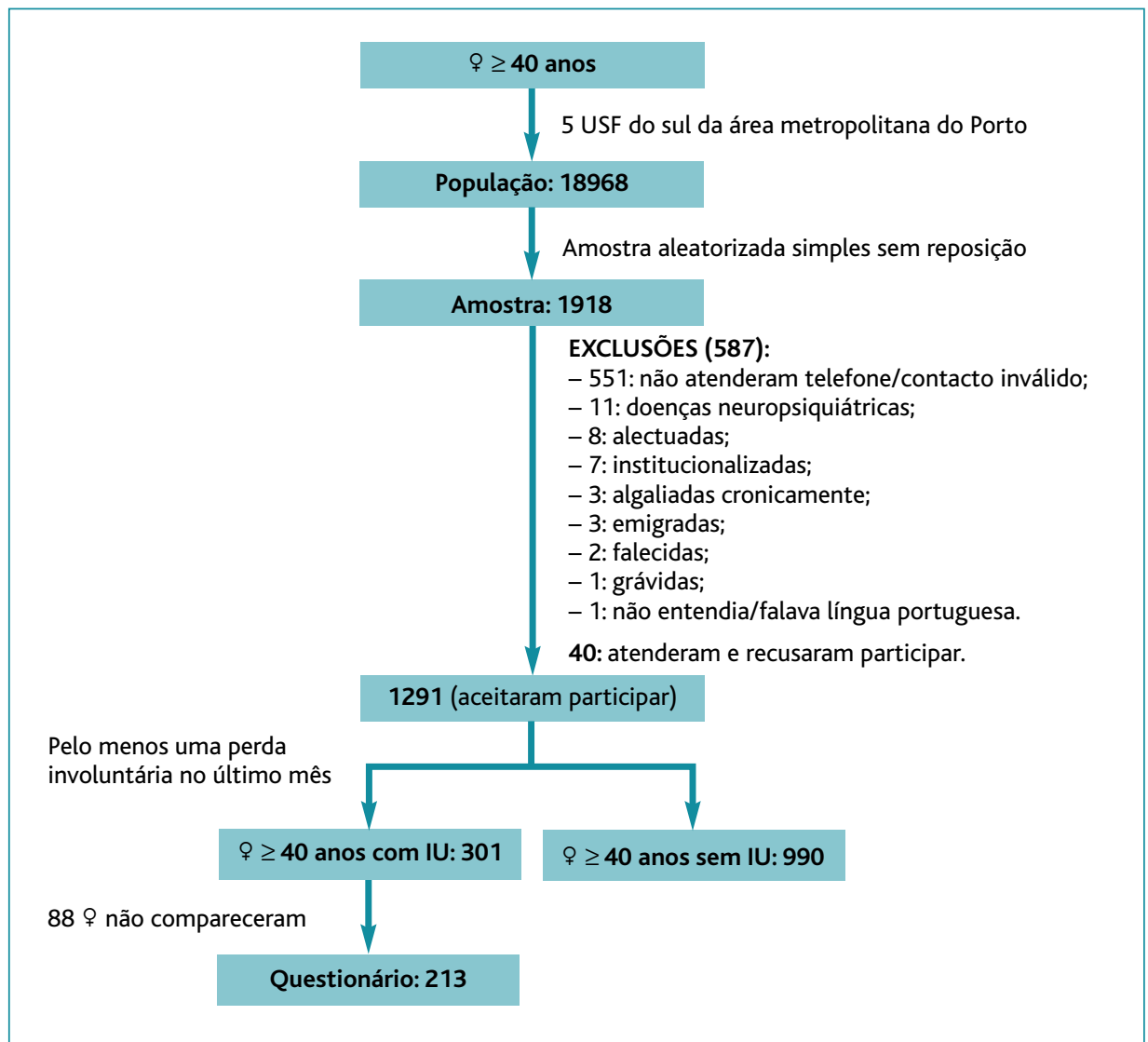

Figura 1. Processo de seleção da amostragem das mulheres incluídas no estudo. IU: incontinência urinária.

de urina, no último mês». Assim foi considerado pelos autores este limite de um mês para uma maior objetivação temporal. ${ }^{1}$ Enquanto, a IU de esforço correspondia aos casos com resposta afirmativa à questão «A perda de urina aconteceu sem vontade de urinar e quando fazia algum esforço, como por exemplo caminhar, tossir, rir, fazer a cama?», perante uma IU de urgência/imperiosidade foi dada uma resposta afirmativa à pergunta «A perda de urina aconteceu quando teve vontade repentina de urinar?» e nos casos de IU mista foi dada resposta afirmativa a ambas as premissas anteriores. ${ }^{14}$

As variáveis independentes estudadas foram: idade, escolaridade, estado civil, índice de massa corporal (IMC), paridade, tipo e tempo de IU e abordagem da IU com e pelo MF.

A referência da IU ao MF como um problema de saúde correspondia à resposta à pergunta «Alguma vez falou com o seu médico de família sobre a perda de uri- na?», enquanto o estabelecimento de orientação para o tratamento da IU dizia respeito à perceção do doente de ter recebido de alguma orientação específica para o tratamento, incluindo: exercícios do pavimento pélvico, tratamento farmacológico, referenciação a consulta de especialidade ou referenciação a tratamento de fisioterapia.

A variável dependente estudada, o impacto da IU na QdV, foi obtida através do questionário CONTILIFE@, Questionnaired'évaluation de la Qualitéde Vie liée à l'incontinence urinaire de la fem$m e "{ }^{15}$ Este foi construído com a finalidade de avaliar o impacto da IU na QdV das mulheres e permite a sua quantificação tendo em conta 6 dimensões distintas: atividades quotidianas, situações de esforço, autoimagem, impacto emocional, sexualidade equalidade de vida em geral.Éum instrumento adaptado e validado para a população portuguesa e consiste em 28 itens, com uma escala de Likert de cinco ou seis pontos. ${ }^{15}$ Para cada questão existem cinco ou seis opções de respostas possíveis, que nalguns itens se relacionam com indicadores de frequência (nunca, raramente, por vezes, frequentemente e sempre) e noutros com indicadores de intensidade (não, pouco, moderadamente, muito e muitíssimo). A análise dos dados foi efetuada calculando um score por dimensão e um score global, de acordo com as normas do respetivo questionário. $\mathrm{O}$ score obtido é diretamente proporcional à $\mathrm{QdV} .^{15}$

Assim, o questionário aplicado (anexo I) consistiu numa primeira parte efetuada pelos autores com o objetivo de colher as variáveis já descritas e numa segunda parte constituída pelo CONTILIFE@.

A análise estatística descritiva e inferencial foi realizada pelo programa SPSS $20.0 \AA$, onde se determinou a 
normalidade da distribuição da amostra pelo teste $\mathrm{Kol}$ mogorov-Smirnov e a homogeneidade de variâncias pelo teste de Levene. Perante uma distribuição normal dos dados, os testes paramétricos usados foram o coeficiente de correlação de Pearson para estudo da associação linear entre variáveis e o teste t Student para comparação de média entre dois grupos. O método não paramétrico utilizado foi o de Spearman. Adotou-se um nível de significância para um $p \leq 0,05$.

O protocolo deste trabalho foi aprovado pelos ACeS envolvidos e pela Comissão de Ética para a Saúde da Administração Regional de Saúde do Norte (Parecer $n^{\circ}$ 11/2012, de 3 de julho de 2012).

\section{RESULTADOS}

Da amostra de 1.918 mulheres, 587 foram excluídas por aplicação dos respetivos critérios e 40 recusaram participar quando foram contactadas por telefone (taxa de resposta de 97\%). Das 1.291 que concordaram participar, 301 afirmaram terem tido pelo menos uma perda de urina involuntária no último mês, sendo a prevalência de IU nas mulheres inquiridas de 23,3\% (95\% IC 22,6-24,0\%). Destas 301 mulheres com IU, 88 (29,2\%) não compareceram na unidade funcional para responder ao questionário na data prevista, concluindo o estudo 213 mulheres com IU (Figura 1).

A idade média das mulheres com IU inquiridas foi de $59,6(D P \pm 1,60)$ anos, $69,0 \%$ (147) era casada e $77,5 \%$ (165) tiveram entre 1 a 3 filhos. Quanto ao nível educacional, $48,4 \%$ (103) tinham o primeiro ciclo completo. $\mathrm{Na}$ amostra em estudo, o IMC médio foi de 28,7 $(D P \pm 0,82)$. Em 84,0\% das inquiridas, foi demonstrado terem IU há mais de um ano, sendo que o tipo de IU, na grande maioria (81,6\%), é mista ou de esforço. As características das mulheres inquiridas estão descritas no Quadro I.

Segundo as mulheres, a IU interferiu de forma moderada na QdV global (avaliada pelo score total), com um resultado médio de 6,7 em 10. Em média, os domínios mais afetados foram as situações de esforço $(6,2 \mathrm{em}$ 10), auto-imagem (6,2 em 10) e impacto emocional (5.9 em 10). Para todas as áreas avaliadas pelo CONTILIFEC, pelo menos $71,8 \%$ das mulheres admite que a IU interfere moderadamente ou mais na sua QdV. A autoimagem é a área onde existe um maior impacto $96,7 \%$ das mulheres mencionam um impacto moderado ou superior.

\begin{tabular}{|c|c|c|}
\hline \multicolumn{3}{|c|}{$\begin{array}{l}\text { QUADRO I. Características das mulheres participantes } \\
\text { com incontinência urinária. }\end{array}$} \\
\hline & média & DP \\
\hline Idade (anos) & 59,6 & 11,71 \\
\hline \multirow{2}{*}{ IMC } & 28,7 & 6,03 \\
\hline & $n$ & $\%$ \\
\hline \multicolumn{3}{|l|}{ Escolaridade } \\
\hline Sem estudos & 20 & 9,4 \\
\hline 1. ${ }^{\circ}$ Ciclo & 103 & 48,4 \\
\hline 2. ${ }^{\circ}$ Ciclo & 28 & 13,1 \\
\hline 3. Ciclo & 24 & 11,3 \\
\hline Secundário & 18 & 8,5 \\
\hline Ensino superior & 20 & 9,4 \\
\hline \multicolumn{3}{|l|}{ Estado civil } \\
\hline Solteira & 7 & 3,3 \\
\hline Casada & 147 & 69,0 \\
\hline Viúva & 33 & 15,5 \\
\hline Outro & 26 & 12,2 \\
\hline \multicolumn{3}{|l|}{ Partos prévios } \\
\hline 0 & 15 & 7,0 \\
\hline $1-3$ & 165 & 77,5 \\
\hline 4 ou mais & 33 & 15,5 \\
\hline \multicolumn{3}{|l|}{ Tipo de IU } \\
\hline Urgência & 39 & 18,3 \\
\hline Esforço & 87 & 40,8 \\
\hline Mista & 87 & 40,8 \\
\hline \multicolumn{3}{|l|}{ Tempo de IU } \\
\hline$\leq 1$ anos & 34 & 16,0 \\
\hline $1-5$ anos & 96 & 45,0 \\
\hline$\geq 5$ anos & 83 & 39,0 \\
\hline \multicolumn{3}{|l|}{ Referência ao MF } \\
\hline $\operatorname{Sim}$ & 80 & 37,6 \\
\hline Não & 133 & 62,4 \\
\hline \multicolumn{3}{|l|}{ Orientação pelo MF } \\
\hline Sim & 51 & 63,8 \\
\hline Não & 29 & 36,2 \\
\hline
\end{tabular}

DP desvio-padrão; IMC: índice de massa corporal; IU: incontinência urinária; MF: médico de família.

O impacto na QdV é independente do tipo de IU para a QdV em geral e nos restantes domínios, exceto para a dimensão «situações de esforço», em que a QdV é me- 


\begin{tabular}{|c|c|c|c|c|}
\hline \multicolumn{5}{|c|}{$\begin{array}{l}\text { QUADRO II. Impacto na qualidade de vida de acordo com o tipo de } \\
\text { incontinência urinária. }\end{array}$} \\
\hline \multirow{2}{*}{$\begin{array}{l}\text { Dimensões } \\
\text { Contilife@ }\end{array}$} & \multicolumn{2}{|c|}{ Média $(0-10)$} & \multirow{2}{*}{$t$-value* } & \multirow{2}{*}{$p$-value* } \\
\hline & $\begin{array}{l}\text { IU Esforço } \\
(n=87)\end{array}$ & $\begin{array}{l}\text { IU Urgência } \\
(n=39)\end{array}$ & & \\
\hline Atividades diárias & 7,71 & 6,95 & $-2,04$ & 0,04 \\
\hline Situações de esforço & 5,56 & 8,46 & 7,28 & $<0,001$ \\
\hline Autoimagem & 6,41 & 6,05 & $-0,74$ & 0,46 \\
\hline Impacto emocional & 6,19 & 5,48 & $-1,34$ & 0,18 \\
\hline Sexualidade & 8,24 & 8,78 & 0,98 & 0,33 \\
\hline QdV geral & 6,77 & 6,81 & 0,08 & 0,94 \\
\hline
\end{tabular}

* Teste t Student

IU: incontinência urinária; QdV: qualidade de vida.

nor na IU de esforço (5,56 \pm 0,46 IC 95\%, versus $8,46 \pm$ 0,58 IC 95\% na IU de urgência). Por sua vez, a QdV na dimensão «atividades diárias» é menor na IU de urgência $(6,95 \pm 0,70$ IC $95 \%$, versus $7,71 \pm 0,38$ IC $95 \%$ na IU de esforço). Estas diferenças encontradas são estatisticamente significativas $(p<0,05)$ - Quadro II.

Relativamente à correlação das restantes variáveis estudadas com o impacto na QdV, os Quadros III e IV sumariam os resultados encontrados. No que diz respeito à variável idade, embora a nível do score total não haja diferenças significativas, na avaliação isolada das dimensões do CONTILIFE@ foi encontrada uma relação direta ténue (15\%), estatisticamente significativa ( $p=$ 0 , 29), entre idade e QdV na dimensão «situações de esforço». De igual forma, há uma relação direta discreta (de $23 \%)$, estatisticamente significativa $(p=0,001)$, entre idade e QdV na dimensão «sexualidade». Assim sendo, a idade associou-se a um impacto negativo na QdV, no que diz respeito às situações de esforço e à sexualidade.

A escolaridade não se associa a impacto significativo na $\mathrm{QdV}$ total nesta amostra $(p=0,06)$. No entanto, foi identificada uma associaçãosignificativa negativa entre a escolaridade e a dimensão "atividades diárias» $(p<0,001)$, ou seja, quanto maior a escolaridade, menor a QdV neste domínio.

A QdV da mulher com IU depende inversamente do IMC ( $p=0,038)$, ou seja, quanto maior o IMC, menor a QdV, sendo esta observação sobretudo na dimensão «atividades diárias» $(p=0,004)$.
Quanto ao estado civil e à duração da IU, não foi encontrada associação estatisticamente significativa com a QdV, quer pelo score total quer em qualquer das dimensões estudadas.

Ter filhos associou-se de forma negativa à QdV total em comparação com as mulheres que nunca tiveram filhos, de forma estatisticamente significativa $(p=0,021)$, com maior expressão a nível das dimensões «situações de esforço» $(p=0,004) \mathrm{e}$ «sexualidade» $(p<0,001)$.

Das $37,6 \%$ de mulheres com IU que abordaram a questão da IU com o seu MF, 63,8\% teve uma orientação. O impacto da IU na QdV associou-se a diferenças estatisticamente significativas na referência deste problema de saúde ao MF. As mulheres que abordaram as perdas de urina com o MF tinham, em média, menor QdV total e em todas as dimensões estudadas $(p<0,05)$.

Relativamente à existência ou não de orientação para o problema da IU, não foi encontrada nenhuma relação com significância estatística em qualquer dos domínios estudados ou pelo score total.

\section{DISCUSSÃO}

Este estudo identificou uma prevalência de IU na população portuguesa de $23,3 \%$, numa amostra de 1.291 mulheres e foi, tanto quanto sabemos, o primeiro estudo nacional que avaliou o impacto desta patologia na QdV através de uma escala validada para a população portuguesa. De uma forma global, as mulheres inquiridas tiveram um prejuízo significativo na sua QdV total (6,7 em 10), referindo impacto nas suas várias dimensões, incluindo sexualidade ( $8,5 \mathrm{em} 10)$, autoimagem (6,2 em 10) e impacto emocional (5,9 em 10).

Este estudo não limitou a amostragem às mulheres frequentadoras dos CSP, mas antes a todas as mulheres inscritas neste nível de cuidados de saúde. No que diz respeito à prevalência de IU, foi possível obter uma taxa de resposta significativa (taxa de não resposta de cerca de $3 \%$ ).

Um dos fatores que influencia a prevalência de IU é a idade das mulheres incluídas nos estudos. Escolhemos este grupo etário (40 anos ou mais) uma vez que as referências bibliográficas analisadas apresentam os 40 anos como o ponto limiar a partir do qual a prevalência de IU aumenta exponencialmente.,14-16 


\begin{tabular}{|c|c|c|}
\hline \multicolumn{3}{|c|}{$\begin{array}{l}\text { QUADRO III. Impacto da incontinência urinária na } \\
\text { qualidade de vida e sua associação com idade, } \\
\text { escolaridade e índice de massa corporal. }\end{array}$} \\
\hline & Correlação & $p$-value* \\
\hline \multicolumn{3}{|l|}{ Idade } \\
\hline SCORE TOTAL & 0,01 & 0.89 \\
\hline Atividades diárias & $-0,08$ & 2,24 \\
\hline Situações de esforço & 0,15 & 0,03 \\
\hline Autoimagem & $-0,02$ & 0,81 \\
\hline Impacto emocional & $-0,09$ & 0,17 \\
\hline Sexualidade & 0,23 & $<0,001$ \\
\hline \multicolumn{3}{|l|}{ Escolaridade } \\
\hline SCORE TOTAL & 0,13 & 0,06 \\
\hline Atividades diárias & $-0,30$ & $<0,001$ \\
\hline Situações de esforço & 0,01 & 0,92 \\
\hline Autoimagem & 0,13 & 0,06 \\
\hline Impacto emocional & $-0,11$ & 0,10 \\
\hline Sexualidade & $-0,01$ & 0,88 \\
\hline \multicolumn{3}{|l|}{ IMC } \\
\hline SCORE TOTAL & $-0,14$ & 0,04 \\
\hline Atividades diárias & $-0,12$ & 0,004 \\
\hline Situações de esforço & $-0,06$ & 0,40 \\
\hline Autoimagem & $-0,11$ & 0,11 \\
\hline Impacto emocional & $-0,07$ & 0,29 \\
\hline Sexualidade & $-0,12$ & 0,08 \\
\hline
\end{tabular}

IMC: índice de massa corporal

*Correlação de Spearman (Idade e IMC); Correlação de Pearson (Escolaridade)

Outro aspeto a ter em conta na prevalência da IU é a sua própria definição e a validade do instrumento de recolha dos dados. Como já foi mencionado, a International Continence Society criou uma definição para ser usada nos estudos epidemiológicos, que foi aquela adotada pelos autores. Existem questionários validados para várias populações que permitem a definição e classificação da IU. No entanto, para a população portuguesa ainda nenhum questionário foi validado. Embora existam vários questionários traduzidos na língua portuguesa, estão apenas validados para a população brasileira. Foi por este motivo que os autores preferiram construir as perguntas que iriam determinar a classificação de IU, embora isso possa constituir uma limitação do estudo. Estas perguntas foram baseadas no estudo de Sandvik de 1995 e no estudo EPICONT de 2003. ${ }^{14,19} \mathrm{De}$

\begin{tabular}{|c|c|c|}
\hline \multicolumn{3}{|c|}{$\begin{array}{l}\text { QUADRO IV. Impacto da incontinência urinária na } \\
\text { qualidade de vida e sua associação com estado civil, } \\
\text { duração da incontinência, paridade e abordagem com o } \\
\text { médico de família. }\end{array}$} \\
\hline & t-value & p-value \\
\hline \multicolumn{3}{|l|}{ Estado civilt } \\
\hline SCORE TOTAL & $-0,18$ & 0,64 \\
\hline Atividades diárias & $-0,65$ & 0,52 \\
\hline Situações de esforço & 0,84 & 0,4 \\
\hline Autoimagem & $-1,47$ & 0,14 \\
\hline Impacto emocional & $-0,3$ & 0,73 \\
\hline Sexualidade & 0,8 & 0,42 \\
\hline \multicolumn{3}{|l|}{ Duração de IU ¥ } \\
\hline SCORE TOTAL & 1,52 & 0,13 \\
\hline Atividades diárias & 1,14 & 0,26 \\
\hline Situações de esforço & 1,04 & 0,3 \\
\hline Autoimagem & 1,19 & 0,24 \\
\hline Impacto emocional & 1,72 & 0,09 \\
\hline Sexualidade & 0,74 & 0,46 \\
\hline \multicolumn{3}{|l|}{ Partos prévios ${ }^{\S}$} \\
\hline SCORE TOTAL & 2,54 & 0,02 \\
\hline Atividades diárias & 1,99 & 0,06 \\
\hline Situações de esforço & 3,3 & 0,004 \\
\hline Autoimagem & 1,28 & 0,21 \\
\hline Impacto emocional & 1,75 & 0,10 \\
\hline Sexualidade & 6,15 & $<0,001$ \\
\hline \multicolumn{3}{|l|}{ Referência ao MF } \\
\hline SCORE TOTAL & $-4,67$ & $<0,001$ \\
\hline Atividades diárias & $-4,6$ & $<0,001$ \\
\hline Situações de esforço & $-3,12$ & 0,02 \\
\hline Autoimagem & $-3,88$ & $<0,001$ \\
\hline Impacto emocional & $-3,92$ & $<0,001$ \\
\hline Sexualidade & $-2,36$ & 0,02 \\
\hline \multicolumn{3}{|l|}{ Orientação pelo MF } \\
\hline SCORE TOTAL & 0,85 & 0,4 \\
\hline Atividades diárias & 1,14 & 0,26 \\
\hline Situações de esforço & 0,35 & 0,73 \\
\hline Autoimagem & 1,16 & 0,25 \\
\hline Impacto emocional & 0,51 & 0,61 \\
\hline Sexualidade & $-0,46$ & 0,65 \\
\hline
\end{tabular}

IU: incontinência urinária; MF: médico de família; $¥$ Entre $1-5$ anos vs $>5$ anos; † Casada vs não casada; $§$ Presença de partos prévios vs ausência de partos prévios

* Teste t Student 
acordo com a International Continence Society, a estimativa da prevalência para cada tipo de IU é sensível à resposta de questões que contêm a definição de IU de esforço e de urgência; de facto, os autores não encontraram um valor específico de sensibilidade/especificidade para esta pergunta, porém a mesma foi usada nos dois estudos de prevalência de IU em Portugal. ${ }^{24,25}$ Sabe-se também que a própria metodologia de obtenção dos dados pode influenciar a prevalência, nomeadamente, através da alusão à patologia na introdução ao estudo e se a resposta aos questionários é feita por entrevista, via telefónica ou por autopreenchimento. ${ }^{20,21}$ No presente estudo, a questão usada para a determinação da prevalência foi feita telefonicamente, o que pode condicionar, de facto, uma menor prevalência de IU, à luz destas fontes bibliográficas. Porém, a introdução efetuada aquando do telefonema não mencionava o nome da patologia que estava a ser estudada.

Quando comparado com outros estudos, as taxas de prevalência de IU encontradas são muito variadas (5$-69 \%)$ por diversos motivos, nomeadamente pelo método de amostragem, pela idade das mulheres incluídas nos estudos, pela metodologia e ferramentas usadas para obtenção dos dados, pela própria definição de IU e dos seus subtipos, bem como a inclusão ou não do grau de severidade da IU. .-5,16-18 $^{-5}$

Comparativamente a estudos europeus que usaram como amostra mulheres com mais de 40 anos de idade, existem várias discrepâncias. Um estudo italiano de Bortolotti et al., a nível dos CSP, com uma amostra de 2.767 mulheres e com uma metodologia semelhante, por contacto telefónico, mostrou uma prevalência de IU inferior de $11 \%$. Porém, a perda de urina tinha ocorrido no último ano, o que alarga o horizonte temporal da ocorrência de IU comparativamente ao nosso estudo, que apenas se direcionava a IU no último mês..$^{22}$ Fatores que possam justificar a diferença de prevalência poderão estar relacionados com a cultura e aspetos sociais (que poderão influenciar a resposta). Por outro lado, diferenças nos fatores sociodemográficos, como a idade e o IMC, poderão contribuir para a diferença nos resultados.

Um estudo populacional dinamarquês de 2000 (Moller et al.) que envolveu 4.000 mulheres, das quais 2.864 foram incluídas, apresenta uma prevalência de IU inferior $(16,1 \%) .{ }^{23}$ Não obstante, este estudo tinha uma metodologia diferente, quer na definição da IU, quer na obtenção dos dados, que ocorreu através do envio de questionários de autopreenchimento por correio eletrónico. Além disso, o estudo excluiu mulheres com idade superior a 60 anos, o que pode ter condicionado diminuição significativa da prevalência (atendendo ao seu aumento com a idade). De sublinhar que este estudo pode ter sofrido viés de seleção pela taxa de não resposta de $30 \%$.

A nível nacional foram encontrados dois estudos de prevalência. Um, de 2005, cuja prevalência determinada foi de $35,2 \%$ e que incluiu 400 mulheres com 40 ou mais anos de idade que recorreram a uma consulta com $\mathrm{o} \mathrm{MF}$, num determinado período. ${ }^{24} \mathrm{O}$ facto de apenas incluir mulheres frequentadoras da consulta de Medicina Geral e Familiar poderá ter contribuído para uma maior prevalência. Além disso, foi utilizado um inquérito de autopreenchimento que, tal como referido anteriormente, pode condicionar maiores taxas de prevalência. Este estudo também confirmou que apenas um terço das mulheres aborda o problema com o seu médico.

Por seu lado, o estudo populacional nacional do Serviço de Higiene e Epidemiologia da Faculdade de Medicina do Porto, publicado em 2008, que usou a mesma definição de IU, o contacto por via telefónica e a mesma idade, obteve uma prevalência sensivelmente aproximada $(21,4 \%$, para $\mathrm{n}=1.483)$. De salientar que, neste estudo, o tamanho amostral foi relativamente superior $(\mathrm{n}=1.918)^{25}$ e que apenas $4,9 \%$ dos casos de IU estavam diagnosticados.

No que diz respeito à QdV, ainda existem poucos estudos publicados, a nível internacional, que usam o CONTILIFE@ para avaliação de QdV. Um estudo francês de Saadoun et al. tenta correlacionar a gravidade da IU com impacto na QdV e mostra que, independentemente da severidade da IU, todas as dimensões do CONTILIFE@ estão negativamente afetadas..$^{26}$

A avaliação do impacto na QdV acrescenta informação importante, uma vez que os danos causados pela IU diferem entre as mulheres e a avaliação isolada dos sintomas pode ser insuficiente para que o médico apreenda a dimensão do problema. Pese embora a identificação da IU e posterior caracterização da QdV da mulher poder identificar casos não relatados de transtorno significativo na vida diária, há que considerar também que a aplicação destas escalas pode traduzir-se na sobrevalorização de uma situação que, sob o 
ponto de vista da utente, não a afeta na sua definição de estar saudável. O impacto da IU na QdV depende, por exemplo, do grau de aceitação e adaptação à IU. Mais do que avaliar a doença, o MF tem nas suas competências a capacidade de avaliar a dolência, ou seja o sofrimento e repercussões biopsicossociais da IU, colocando a pessoa no centro da sua ação orientadora. Um momento oportuno para a abordagem desta problemática é aquando da realização de consultas de planeamento familiar ou rastreio do cancro do colo do útero. A abordagem desta problemática poderia igualmente ocorrer através de intervenções comunitárias, como distribuição de panfletos informativos e realização de sessões de educação para a saúde. A perceção da paciente sobre a sua IU pode ser um fator fulcral para ela procurar ajuda ou, então, desvalorizar o problema e não o abordar com o médico.

Quanto à relação entre a QdV e o tipo de incontinência, como verificado no estudo realizado por Dedicação AC et al., observou-se que a QdV é independente do tipo de incontinência. ${ }^{27}$

Porém, a literatura não é unânime a este nível, devido a diferenças metodológicas e ao tipo de questionário para avaliação do impacto (questionário específicos para a IU versus questionários genéricos de avaliação da QdV). Existem trabalhos que apontam para que a IU mista terá um maior impacto na QdV, uma vez que a coexistência das duas formas poderá ter um efeito aditivo no agravamento da $\mathrm{QdV} \cdot{ }^{19,28-33}$ No entanto existe um estudo de Coyne et al. que ressalva que a pior QdV nas mulheres com IU mista se deve ao componente de urgência e que há um maior impacto na QdV das mulheres com IU mista e de urgência. ${ }^{34}$ Noutros trabalhos verificou-se uma associação entre a IU de urgência e uma pior QdV, no entanto as amostras eram pequenas e os questionários careciam de sensibilidade. ${ }^{35,36}$ Os autores salientam que na IU urgência, pela sua fisiopatologia, os sintomas são muito mais frequentes e imprevisíveis, com um consequente maior impacto na QdV. Por seu lado, a IU de esforço acontece perante determinados eventos que podem ser prevenidos, como por exemplo evitar exercício físico intenso ou pegar em objetos pesados.

O impacto da IU na QdV aumenta com o índice de massa corporal e com a paridade. Estes achados são consistentes com outros estudos similares. ${ }^{31,33,37-40}$ Uma explicação para que maior IMC e a paridade estejam associados a uma pior QdV pode estar relacionada com o facto de ambas as variáveis serem consideradas fatores de risco para a IU e, adicionalmente, pode estar presente um fator de confundimento devido a não ter sido estudada a severidade da IU, que pode ser maior em mulheres com maior IMC e que tiveram filhos.

No domínio das actividades quotidianas, a associação de uma pior QdV em mulheres instruídas pode ser explicada por estas estarem mais informadas sobre a IU e valorizarem mais o impacto da doença nas suas actividades, em comparação com mulheres menos instruídas.

Das mulheres com IU, $38 \%$ falaram do problema ao seu MF, o que é superior ao encontrado no estudo EPICONT e no estudo de Altawell et al., no entanto é semelhante ao encontrado no estudo português de Moura. ${ }^{19,24,41}$ As razões para a não abordagem do problema com o MF já foram abordadas na introdução.

À semelhança de outros trabalhos, as mulheres que falam do problema ao médico de família têm uma pior QdV em comparação com as que não falam. .0,41,42 $^{4}$

Os resultados deste estudo devem ser interpretados com precaução devido a algumas limitações. No que diz respeito ao objetivo primário (prevalência), para além de existir variabilidade significativa entre os estudos, os resultados podem não ser generalizáveis para a população portuguesa, dado o local de estudo se ter centrado numa área pequena do país e fundamentalmente urbana. Porém, o facto dos resultados encontrados neste estudo serem semelhantes a um estudo prévio realizado a nível nacional, com metodologia semelhante, sugere consistência nos mesmos. No que se refere aos objetivos secundários, poderá haver potencial para viés de seleção pelo facto de parte significativa $(29,2 \%)$ das mulheres não terem comparecido nas unidades para a realização do inquérito CONTILIFE@.

Em conclusão, embora tenha uma prevalência alta na população feminina, apenas cerca de um terço das mulheres com IU aborda o problema com o MF e, destas, um terço não tem qualquer tipo de orientação. A IU tem um impacto negativo na QdV geral das mulheres. As situações de esforço, autoimagem e impacto emocional são as dimensões mais afetadas, mas também a nível das atividades diárias e sexualidade. O impacto da IU na QdV está relacionado com o IMC, número de partos prévios e com a referência do problema ao MF. As- 
sim, o MF deve estar sensibilizado para o problema da IU e atualizado relativamente às opções terapêuticas e possíveis orientações no sentido de minimizar o impacto da IU na QdV.

Futuros estudos de investigação nesta área deverão procurar obter uma amostra que possa ser generalizável à população portuguesa. Por outro lado, seria interessante apurar, de forma prospetiva, o impacto das intervenções na QdV das mulheres com IU.

\section{REFERÊNCIAS BIBLIOGRÁFICAS:}

1. Milson I. Epidemiology of urinary and faecal incontinence and pelvic organ prolapsed. In: Abrams P, Cardozo L, Khoury S, Wein A, editors. Incontinence. 4th ed. Bristol: International Continence Society; 2009 [acedido em 01/06/2011]. Disponível em

2. Santos VV. Incontinência urinária feminina. Rev Port Clin Geral 2003 Jul-Ago; 19 (4): 369-75.

3. Wennberg AL, Molander U, Fall M, Edlund C, Peeker R, Milsom I. A longitudinal population-based survey of urinary incontinence, overactive bladder, and other lower urinary tract symptoms in women. Eur Urol 2009 Apr; 55 (4): 783-91.

4. Trüroff JW, Abrams P, Andersson KE, Artibani W, Chapple CR, Drake MJ, et al. EAU guidelines on urinary incontinence. Eur Urol 2011 Mar; 59 (3): 387-400.

5. Farage MA, Miller KW, Berardesca E, Maibach HI. Psychosocial and societal burden of incontinence in the aged population: a review. Arch Gynecol Obstet 2008 Apr; 277 (4): 285-90.

6. Yip SK, Cardozo L. Psychological morbidity and female urinary incontinence. Best Pract Res Clin Obstet Gynaecol 2007 Apr; 21 (2): 321-9.

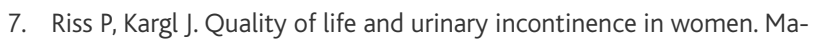
turitas 2011 Feb; 68 (2): 137-42.

8. Broome BA. The impact of urinary incontinence on self-efficacy and quality of life. Health Qual Life Outcomes 2003 Aug 22; 1: 35.

9. Higa R, Lopes MH, Turato ER. Psychocultural meanings of urinary incontinence in women: a review. Rev Lat Am Enfermagem 2008 Jul-Aug; 16 (4): 779-86.

10. Corcos J, Beaulieu S, Donovan J, Naughton M, Gotoh M; Symptom Quality of Life Assessment Committee of the First International Consultation on Incontinence. Quality of life assessment in men and women with urinary incontinence. J Urol 2002 Sep; 168 (3): 896-905.

11. Schaw C, Tansey R, Jackson C, Hyde C, Allan R. Barriers to help seeking in people with urinary symptoms. Fam Pract 2001 Feb; 18 (1): 48-52.

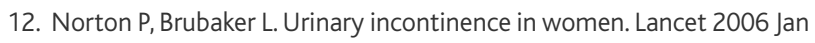
7; 367 (9504): 57-67.

13. Higa R, Lopes $M H$, dos Reis MJ. Fatores de risco para incontinência urinária na mulher. Rev Esc Enferm USP 2008 Mar; 42 (1): 187-92.

14. Sandvik H, Hunskaar S, Vanvik A, Bratt H, Seim A, Hermstad R. Diagnostic classification of female urinary incontinence: an epidemiological survey corrected for validity. J Clin Epidemiol 1995 Mar; 48 (3): 33943.

15. Paiva C, Silva AP, Robalo L. Contributo para a adaptação e validação do instrumento de medida "Questionário de avaliação da qualidade de vida em mulheres com incontinência urinária (Contilife@) para a população portuguesa feminina. Ifisionline 2010; 1: 7-17.

16. Cheater FM, Castleden CM. Epidemiology and classification of urinary incontinence. Ballieres Best Pract Res: Clin Obstet \& Gynaecol 2000 Apr; 14 (2): 183-205.

17. Cerruto MA, D'Elia C, Aloisi A, Fabrello M, Artibani W. Prevalence, incidence and obstetric factors' impact on female urinary incontinence in Europe: a systematic review. Urol Int 2013; 90 (1): 1-9.

18. Hunskaar S, Burgio K, Diokno A, Herzog AR, Hjalmås K, Lapitan MC. Epidemiology and natural history of urinary Incontinence in women. Urology 2003 Oct; 62 (4 Suppl 1): 16-23.

19. Hannestad YS, Rortveit G, Sandvik H, Hunskaar S. A community based epidemiologic survey of female urinary incontinence: The Norwegian EPICONT study. Epidemiology of incontinence in the county of NordTrøndelag. J Clin Epidemiol 2000 Nov; 53 (11): 1150-7.

20. Fultz $\mathrm{NH}$, Herzog AR. Prevalence of urinary incontinence in middle-aged and older women: a survey-based methodological experiment. J Aging Health 2000 Nov; 12 (4): 459-69.

21. Botlero R, Urquhart DM, Davis SR, Bell RJ. Prevalence and incidence or urinary incontinence in women: review of the literature and investigation of methodological issues. Int J Urol 2008 Mar; 15 (3): 230-4.

22. Bortolotti A, Bernardini B, Colli E, Di Benedetto P, Giocoli Nacci G, et al. Prevalance and risk factors for urinary incontinence in Italy. Eur Urol 2000 Jan; 37 (1): 30-5.

23. Møller LA, Lose $G$, and Jørgensen T. The prevalence and bothersomeness of lower urinary tract symptoms in women 40-60 years of age. Acta Obstet Gynecol Scand 2000 Apr; 79 (4): 298-305.

24. Moura BG. Incontinência urinária feminina. Rev Por Clin Geral. 2005 Jan-Fev; 21 (1): 11-20.

25. Serviço de Higiene e Epidemiologia da Faculdade de Medicina da Universidade do Porto. Prevalência e tratamento de incontinência urinária na população portuguesa não institucionalizada. Porto: FMUP; 2008. Disponível em: http://www.apurologia.pt/pdfs/estud-epidem-incont08.pdf [acedido em 11/11/2013].

26. Saadoun K, Ringa V, Fritel X, Varnoux N, Zins M, Bréart G. Negative impact of urinary incontinence on quality of life, a cross-sectional study among women aged 49-61 years enrolled in the GAZEL cohort. Neurourol Urodyn 2006; 25 (7): 696-702.

27. Dedicação AC, Haddad M, Saldanha ME, Driusso P. Comparação da qualidade de vida nos diferentes tipos de incontinência urinária feminina. Rev Bras Fisioter 2009 Mar-Abr; 13 (2): 116-122.

28. Yalcin I, Patrick DL, Summers K, Kinchen K, Bump RC. Minimal clinically important differences in incontinence quality-of-life scores in stress urinary incontinence. Urology 2006 Jun; 67 (6): 1304-8.

29. Barghouti FF, Yasein NA, Jaber RM, Hatamleh LN, Takhuri AH. Prevalence and risk factors of urinary incontinence among Jordanian women: impact on their life. Health Care Women Int 2013; 34 (11): 1015-23.

30. Shaw C, Gupta RD, Bushnell DM, Assassa RP, Abrams P, Wagg A, et al. The extent and severity of urinary incontinence amongst women in UK GP waiting rooms. Fam Pract 2006 Oct; 23 (5): 497-506.

31. Firdolas F, Onur R, Deveci SE, Rahman S, Sevindik F, Acik Y. Effect of urinary incontinence and its subtypes on quality of life of women in eastern Turkey. Urology 2012 Dec; 80 (6): 1221-6.

32. Frick AC, Huang AJ, Van den Eeden SK, Knight SK, Creasman JM, Yang J, 
et al. Mixed urinary incontinence: greater impact on quality of life. J Urol 2009 Aug; 182 (2): 596-600.

33. Barentsen J, Visser E, Hofstetter H, Maris A, Dekker J, Bock G. Severity, not type, is the main predictor of decreased quality of life in elderly women with urinary incontinence: a population-based study as part of a randomized controlled trial in primary care. Health Qual Life Outcomes 2012 Dec 18; 10: 153.

34. Coyne KS, Zhou Z, Thompson C, Versi E. The impact on health-related quality of life of stress, urge and mixed urinary incontinence. BJU Int 2003 Nov; 92 (7): 731-5.

35. Simeonova Z, Milsom I, Kullendorff AM, Molander U, Bengtsson C. The prevalence of urinary incontinence and its influence on the quality of life in women from an urban Swedish population. Acta Obstet Gynecol Scand 1999 Jul; 78 (6): 546-51.

36. Schimpf MO, Patel M, O'Sullivan DM, Tulikangas PK. Difference in quality of life in women with urge urinary incontinence compared to women with stress urinary incontinence. Int Urogynecol J Pelvic Floor Dysfunct 2009 Jul; 20 (7): 781-6.

37. Melville JL, Katon W, Delaney K, Newton K. Urinary incontinence in US women: a population-based study. Arch Intern Med 2005 Mar 14; 165 (5): 537-42.

38. Smith AL, Wang PC, Anger JT, Mangione CM, Trejo L, Rodríguez LV, et al. Correlates of urinary incontinence in community-dwelling older Latinos. J Am Geriatr Soc 2010 Jun; 58 (6): 1170-6.

39. Horng SS, Huang N, Wu SI, Fang YT, Chou YJ, Chou P. The epidemiolo- gy of urinary incontinence and its influence on quality of life in Taiwanese middle-aged women. Neurourol Urodynam 2012 Apr; 32 (4): 371-6.

40. Kwon BE, Kim GY, Son YJ, Roh YS, You MA. Quality of life of women with urinary incontinence: a systematic literature review. Int Neurourol J 2010 Oct; 14 (3): 133-8.

41. Altaweel W, Alhatrbi M. Urinary incontinence: prevalence, risk factors, and impact on health related quality of life in Saudi women. Neurourol Urodynam 2012 Jun; 31 (5): 642-5.

42. Guarisi T, Pinto-Neto AM, Osis MJ, Pedro AO, Costa-Paiva LH, Faúndes A. Procura de serviço médico por mulheres com incontinência urinária. Rev Bras Ginecol Obstetr 2011 Ago; 23 (7): 439-43.

\section{CONFLITOS DE INTERESSE}

Os autores declaram não ter conflito de interesses.

\section{ENDEREÇO PARA CORRESPONDÊNCIA}

Ana Isabel Silva

Estrada da Granja, 114 Valbom, 4420-465 GONDOMAR

E-mail: anisabelfs@gmail.com

Recebido em 03-08-2013

Aceite para publicação em 26-11-2013

Artigo escrito ao abrigo do novo acordo ortográfico.

\section{ABSTRACT}

\section{PREVALENCE AND IMPACT OF FEMALE URINARY INCONTINENCE ON QUALITY OF LIFE}

Objectives: Urinary incontinence is a health problem with many negative effects on the quality of life of women. It is often under-diagnosed and untreated. This is a study of the prevalence of urinary incontinence, its impact on quality of life, and its association with demographic factors.

Study Design: analytical cross-sectional

Setting: The family health units of the authors

Participants: A sample of 1918 women over age 40

Methods: The variables studied were age, marital status, education, body mass index, number of children, type of incontinence, duration, referral the problem to the doctor and treatment. The impact on quality of life was assessed using the CONTILIFE $\odot$ questionnaire. The level of significance was set at $p \leq 0.05$.

Results: Of the 1291 women responding (mean age 60 years), 23\% had urinary incontinence. Their quality of life score was 6.7 points out of a possible 10. The impact on quality of life increases $(p \leq 0.05)$ with illiteracy, body mass index, and the number of births. $38 \%$ of women with urinary incontinence have addressed this issue with their doctor. $66 \%$ received either drug treatment or referral. Reporting the problem to the family doctor was associated with greater impact of incontinence on quality of life. $(p<0.001)$

Conclusions: This study found a high prevalence of urinary incontinence in this population with significant impact on quality of life. Only one third of women with urinary incontinence addressed this problem with their doctor and two thirds of these women received drug treatment or referral.

Keywords: Urinary Incontinence; Women; Quality of Life. 


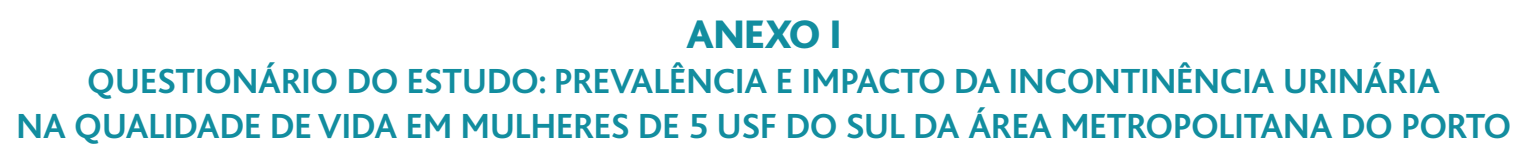

DOENTE $N^{\circ}$

INVESTIGADOR

UNIDADE DE SAÚDE

NUT III

1) Teve alguma perda involuntária de urina (ou seja, sem o seu controlo), no último mês? Sim___ Não (Se não fica excluída do estudo)

2) Em relação, às perdas de urina:

2.1.) A perda de urina aconteceu quando teve vontade repentina de urinar? Sim

Não

2.2.) A perda de urina aconteceu sem vontade de urinar e quando fazia algum esforço, como por exemplo, caminhar, tossir, rir, fazer a cama? Sim

Não_

3) Há quanto tempo começou com essa perda involuntária de urina?

Menos de 1 ano Entre 1 a 5 anos

Mais de 5 anos

4) Alguma vez falou com o seu Médico de Família sobre a perda de urina? Sim

Não

4.1.) SE SIM:

4.1.1.) Teve alguma orientação? Sim

Não

5) Idade: anos

6) Estado Civil? Solteira

Casada Viúva

Outros

7) Escolaridade? Sem Estudos

1. ${ }^{\circ}$ Ciclo

2. ${ }^{\circ}$ Ciclo

3. ${ }^{\circ}$ Ciclo

Ensino Secundário Ensino Superior

8) Peso $\mathrm{Kg}$;

Altura $\mathrm{m} ; \quad \mathrm{IMC}$ $\mathrm{Kg} / \mathrm{m}^{2}$

9) Quantos filhos teve? 0 $1-3$ +4 ou mais 


\section{CONTILIFE@}

\section{ATIVIDADES QUOTIDIANAS}

No decorrer das últimas 4 semanas, as perdas de urina incomodaram-na:

\begin{tabular}{|c|c|c|c|c|c|c|}
\hline & Não Aplicável & Não & Pouco & Moderadamente & Muito & Muitíssimo \\
\hline 1. Quando estava fora de casa? & & 1 & 2 & 3 & 4 & 5 \\
\hline 2. Enquanto conduzia ou viajava de carro? & 0 & 1 & 2 & 3 & 4 & 5 \\
\hline 3. Quando subia ou descia escadas? & & 1 & 2 & 3 & 4 & 5 \\
\hline 4. Ao fazer compras ou outras atividades semelhantes? & & 1 & 2 & 3 & 4 & 5 \\
\hline $\begin{array}{l}\text { 5. Quando esperava numa fila (exemplo: autocarro, } \\
\text { cinema ou supermercado)? }\end{array}$ & & 1 & 2 & 3 & 4 & 5 \\
\hline
\end{tabular}

No decorrer das últimas 4 semanas, devido às perdas de urina, com que frequência:

\begin{tabular}{|l|c|c|c|c|c|}
\hline & Nunca & Raramente & Por Vezes & Frequentemente & Sempre \\
\hline $\begin{array}{l}\text { 6. Teve de interromper o seu trabalho ou as suas atividades } \\
\text { quotidianas? }\end{array}$ & 1 & 2 & 3 & 4 & 5 \\
\hline
\end{tabular}

No decorrer das últimas 4 semanas, devido às perdas de urina, com que frequência:

\begin{tabular}{|l|c|c|c|c|c|}
\hline & Nunca & Raramente & Por Vezes & Frequentemente & Sempre \\
\hline 7. Acordou urinada? & 1 & 2 & 3 & 4 & 5 \\
\hline
\end{tabular}

\section{SITUAÇÕES DE ESFORÇO}

No decorrer das últimas 4 semanas, as perdas de urina incomodaram-na:

\begin{tabular}{|l|c|c|c|c|c|c|}
\hline & Não Aplicável & Não & Pouco & Moderadamente & Muito & Muitíssimo \\
\hline 8. Ao levantar ou a transportar algo pesado? & & 1 & 2 & 3 & 4 & 5 \\
\hline $\begin{array}{l}\text { 9. Ao praticar desporto? (exemplo: caminhadas, dança, } \\
\text { ginástica) }\end{array}$ & 0 & 1 & 2 & 3 & 4 & 5 \\
\hline 10. Quando se assoou, espirrou ou tossiu? & & 1 & 2 & 3 & 4 & 5 \\
\hline 11. Ao rir? & & 1 & 2 & 3 & 4 & 5 \\
\hline
\end{tabular}

\section{AUTO-IMAGEM}

No decorrer das últimas 4 semanas, devido às perdas de urina, com que frequência:

\begin{tabular}{|l|c|c|c|c|c|}
\hline & Nunca & Raramente & Por Vezes & Frequentemente & Sempre \\
\hline 12. Se sentiu menos atraente? & 1 & 2 & 3 & 4 & 5 \\
\hline 13. Teve receio de "cheirar mal"? & 1 & 2 & 3 & 4 & 5 \\
\hline 14. Teve receio de que alguém se apercebesse da sua condição? & 1 & 2 & 3 & 4 & 5 \\
\hline 15. Teve receio de manchar algo na casa de outros ou no trabalho? & 1 & 2 & 3 & 4 \\
\hline 16. Teve de mudar de roupa? & 1 & 2 & 3 & 4 & 5 \\
\hline
\end{tabular}


No decorrer das últimas 4 semanas, apesar das suas perdas de urina, com que frequência:

\begin{tabular}{|l|c|c|c|c|c|}
\hline & Nunca & Raramente & Por Vezes & Frequentemente & Sempre \\
\hline 17. Se sentiu bem consigo mesma? & 1 & 2 & 3 & 4 & 5 \\
\hline
\end{tabular}

No decorrer das últimas 4 semanas, devido às suas perdas de urina:

\begin{tabular}{|c|c|c|c|c|c|c|}
\hline & Eu nunca utilizo proteções & Não & Pouco & Moderadamente & Muito & Muitíssimo \\
\hline $\begin{array}{l}\text { 18. Sentiu-se incomodada por ter de utilizar } \\
\text { proteções? }\end{array}$ & 0 & 1 & 2 & 3 & 4 & 5 \\
\hline
\end{tabular}

\section{IMPACTO EMOCIONAL}

No decorrer das últimas 4 semanas, devido às suas perdas de urina, com que frequência:

\begin{tabular}{|l|c|c|c|c|c|}
\hline & Nunca & Raramente & Por Vezes & Frequentemente & Sempre \\
\hline 19. Se sentiu desmotivada? & 1 & 2 & 3 & 4 & 5 \\
\hline 20. Perdeu a paciência? & 1 & 2 & 3 & 4 & 5 \\
\hline 21. Se sentiu preocupada? & 1 & 2 & 3 & 4 & 5 \\
\hline $\begin{array}{l}\text { 22. Teve a sensação de não conseguir controlar as suas reações? } \\
\begin{array}{l}\text { 23. Os seus problemas urinários constituíram uma obsessão ou um } \\
\text { pavor para si? }\end{array}\end{array}$ & 1 & 2 & 3 & 4 & 5 \\
\hline \begin{tabular}{l} 
24. Teve que programar levar proteções antes de sair? \\
\hline
\end{tabular} & 1 & 2 & 3 & 4 & 5 \\
\hline
\end{tabular}

\section{SEXUALIDADE}

No decorrer das últimas 4 semanas, devido às suas perdas de urina:

\begin{tabular}{|l|c|c|c|c|c|c|}
\hline & Não Aplicável & Não & Pouco & Moderadamente & Muito & Muitíssimo \\
\hline 25. Sentiu-se ansiosa com a ideia de ter relações sexuais? & & 1 & 2 & 3 & 4 & 5 \\
\hline 26. Alterou o seu comportamento sexual? & 0 & 1 & 2 & 3 & 4 & 5 \\
\hline 27. Receou ter perdas durante as relações sexuais? & 0 & 1 & 2 & 3 & 4 & 5 \\
\hline
\end{tabular}

\section{QUALIDADE DE VIDA EM GERAL}

28. Tendo em conta as suas perdas de urina, como avalia atualmente a sua Qualidade de Vida?

\begin{tabular}{|c|c|c|c|c|}
\hline 1 & 2 & 3 & 4 & 5 \\
Má & & & & Excelente \\
\hline
\end{tabular}

